\title{
High Quality NLM Method for Medium Voltage Hybrid Bridge MMC
}

\author{
YE Jiaxiong ${ }^{1}$, *WANG Jikang ${ }^{2}$, PENG Yuanquan ${ }^{1}$, FAN Xinming ${ }^{1}$ and LIU Yijun ${ }^{1}$ \\ ${ }^{1}$ Foshan Power Supply Bureau, Guangdong Power Grid Company, Foshan, 528000, China \\ ${ }^{2}$ Power Supply Company Beijing Sifang Automation Co.,LTD., Beijing, 100085, China
}

\begin{abstract}
Medium voltage hybrid bridge multilevel converter (MMC) usually has a lower number of links. Therefore, the traditional Nearest Level Modulation (NLM) method has the problems of low equivalent switching frequency and poor output quality. And the traditional Carrier Phase-shifted Pulse Width Modulation (CPS-PWM) modulation has problems such as difficulty in hybrid bridge control, difficulty in redundant configuration, and large amount of calculation. In response to the above problems, this paper proposes a new high-quality NLM method. Based on the traditional NLM modulation method, PWM modulation output and module voltage equalization are realized through the time difference between one module input and one module exit in each control cycle. It achieves the purpose of improving the equivalent switching frequency and output power quality, and at the same time has the advantages of the traditional NLM modulation method with small calculation amount, redundant configuration and hybrid bridge modulation. This article describes the three modulation methods and compares their effects. Finally, the theoretical analysis is verified by PSCAD simulation.
\end{abstract}

\section{INTRODUCTION}

In recent years, renewable energy has become the focus of development of all countries in the world. Distributed power generation is an important part of the sustainable development of renewable energy ${ }^{[1]}$. Replacing the traditional AC distribution network with a DC distribution network to achieve access to renewable energy has many advantages. The United States, Japan, and other countries have established demonstration projects for DC distribution networks. ${ }^{[2]-[8]}$

MMC topology has many advantages such as modularization, easy expansion, easy redundancy, etc. [9][10], and is widely used in AC/DC hybrid distribution networks. Scholars at home and abroad have conducted in-depth research on it. At present, its mainstream modulation methods include NLM mode ${ }^{[11]}$ and CPSPWM mode.

The NLM method has the advantages of a small amount of calculation and simple redundancy settings. The main problem is that the modulation output waveform is a step wave, and the switching frequency and the harmonic frequency band caused by the modulation are wider. However, the medium voltage MMC used in the distribution network has a small number of bridge arm modules, and when the traditional NLM modulation method is adopted, the number of output levels is small, the power quality is poor, and the filtering is difficult.

The CPS-PWM method has the advantages of fixed switching frequency and equivalent switching frequency.
But its large amount of calculation, more difficult redundant settings makes it difficult to implement for hybrid bridge MMC.

This article proposes a high-quality NLM modulation method by combining the advantages of the NLM modulation method and the CPS-PWM modulation method. This method has many advantages such as small calculation amount, fixed switching frequency and equivalent switching frequency, significant voltage equalization effect, convenient redundancy design and accurate modulation output, and can be effectively applied to medium voltage $\mathrm{MMC}$ in $\mathrm{AC} / \mathrm{DC}$ hybrid distribution network. This article describes the modulation method in detail and verifies the effectiveness of the modulation by means of PSCAD simulation.

\section{BASIC PRINCIPLES OF HYBRID BRIDGE MMC}

The basic component unit sub-module of MMC has a variety of topological circuits, including half bridge, full bridge and double half bridge, etc. The MMC described in this article adopts a mixed configuration of full-bridge modules and half-bridge modules. Using a suitable ratio of hybrid bridges can complement the advantages of the two topologies. Therefore, hybrid bridge MMC is widely used in engineering projects.

$U_{D C}$ is MMC DC voltage between poles; $u_{j}(j=a, b, c)$ is three-phase $\mathrm{AC}$ voltage. $i_{j}$ is three-phase $\mathrm{AC}$ current.

\footnotetext{
a Corresponding author: author@e-mail.org
} 
$u_{p j}$ is the three-phase upper bridge arm voltage. $i_{p j}$ is the three-phase upper bridge arm current. $u_{j n}$ is the threephase lower bridge arm voltage. $i_{n j}$ is the three-phase lower bridge arm current. According to the topological structure and operating characteristics of $\mathrm{MMC}$, the upper and lower arm voltages of MMC are:
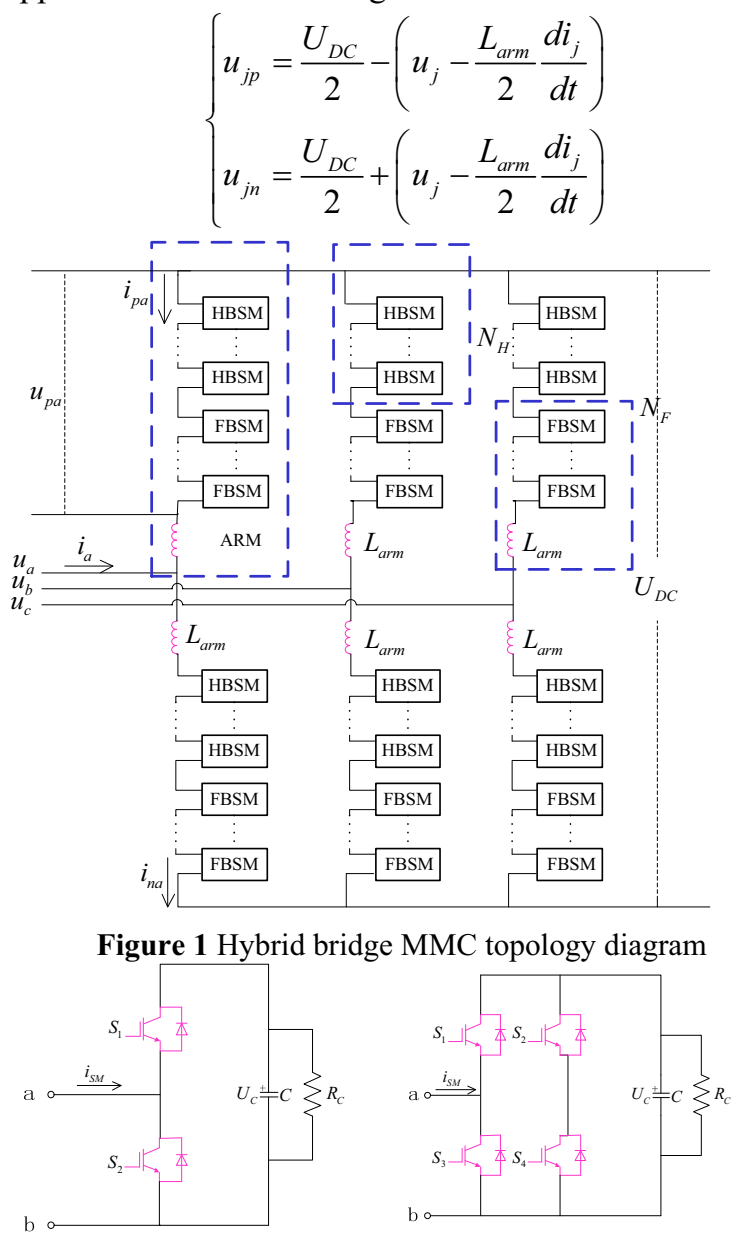

(a) half-bridge sub-module

(b) full-bridge sub-module

Figure 2 MMC module topology diagram

Among them, $U_{D C o u t}$ is a DC voltage output command, $U_{j A C o u t}$ is a $j(j=a, b, c)$ phase AC voltage output command, $U_{\text {jpout }}$ is a $\mathrm{j}$-phase upper bridge arm output voltage command, and $U_{\text {jnout }}$ is a $\mathrm{j}$-phase lower bridge arm output voltage command.

$$
\left\{\begin{array}{l}
U_{\text {jpout }}=\frac{U_{D \text { Cout }}}{2}-U_{j A \text { Cout }} \\
U_{\text {jnout }}=\frac{U_{D \text { Cout }}}{2}+U_{j A \text { Cout }}
\end{array}\right.
$$

The topology of the full bridge and half bridge is shown in Figure 2. According to the analysis, in the unlocked state, according to the different IGBT switches, the halfbridge module has two states: output 0 voltage ( 0 level) and output $+U_{C}$ (positive level); The full-bridge module has three levels: output 0 voltage $(0$ level $)$, output $+U_{C}$ (positive level) and output $-U_{C}$ (negative level). Due to the above differences, the modulation method of the hybrid bridge $\mathrm{MMC}$ is more complicated than the traditional modulation method.

\section{TRADITIONAL NLM MODULATION METHOD AND CPS-PWM MODULATION METHOD}

\subsection{Traditional NLM modulation method}

The NLM modulation method has the advantages of small calculation, low switching frequency, and convenient redundant configuration. It is widely used in the field of high-voltage flexible DC transmission. However, in the field of medium and low-voltage MMC, due to the small number of modules, the equivalent switching frequency of this modulation method is low. This causes problems such as poor output power quality and uneven harmonic frequency distribution. The flowchart of the NLM modulation method taking the Aphase upper bridge arm when the modulated output voltage is positive is shown in Figure 3.

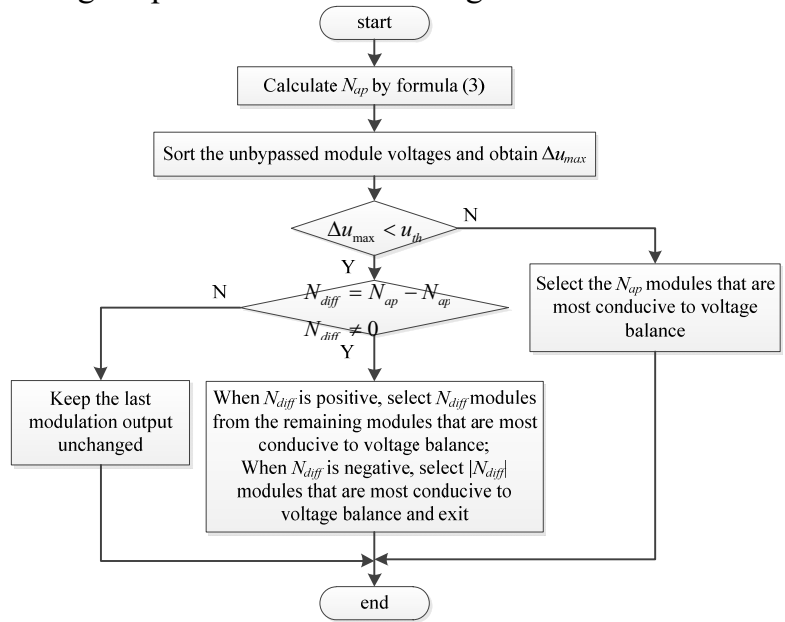

Figure 3 Flow chart of traditional NLM method

When the number of modulation output modules is positive, first calculate the number of modulation modules Nap of the bridge arm through the result of equation (2) as shown in equation (3). Among them um is the rated module voltage. round() is the nearest rounding symbol.

$$
N_{a p}=\operatorname{round}\left(\frac{U_{\text {apout }}}{u_{\mathrm{m}}}\right)
$$

For the MMC configured with redundant modules, the faulty module will be bypassed when the module fails. Next, the modules that are not bypassed are sorted according to voltage and the difference $\Delta u_{\max }$ between the maximum module voltage and the minimum module voltage is obtained. According to the magnitude relationship between $\Delta u_{\max }$ and the sorting threshold $u_{t h}$, it is judged whether the module needs to be fully sorted and output.

When $\Delta u_{\max }>u_{t h}$, perform full sequencing output, and directly select Nap modules that are most conducive to module voltage balance according to the current direction of the bridge arm. When $\Delta u_{\max } \leqslant u_{t h}$, the full sorting output is not performed. At this time, the difference $N_{\text {diff }}$ from the last modulation module number output is calculated according to formula (4). 


$$
N_{\text {apdiff }}=N_{a p}-N_{\text {apold }}
$$

When $N_{\text {apdiff }}=0$, the modulation output remains unchanged from the previous output; when $N_{\text {apdiff }}>0$, according to the current direction of the bridge arm, select $N_{\text {apdiff }}$ modules that are most conducive to voltage balance from the modules that were not put in the last modulation.; When $N_{\text {apdiff }}<0$, according to the current direction of the bridge arm. Select the $\left|N_{\text {apdiff }}\right|$ module that is most unfavorable for voltage equalization from the modules invested last time to exit.

The calculation of NLM modulation is completed above. Among them, when the module output is positive, the bridge arm current $i_{a p}>0$, the input module capacitance is charged, so the module with low input voltage is more conducive to voltage balance; conversely when $i_{a p}<0$, the input module capacitance is discharged, so modules with high voltage are more conducive to voltage balance.

When the output of the modulation module number is negative, only the full-bridge module is considered for output, and the half-bridge modules all exit. The other process is similar to that when the output of the modulation module number is positive, so I won't repeat it here.

\subsection{CPS-PWM modulation method}

The advantage of the CPS-PWM modulation method is that the switching frequency and the output equivalent switching frequency are fixed. In the case of a small number of modules, higher power quality can be obtained, and the harmonic frequency is stable, and the output filter selection is convenient. However, this modulation method has problems such as large amount of calculation, complicated redundant configuration, and too complicated control in the case of a hybrid bridge. The flow chart of the CPS-PWM modulation method in which the modulation output voltage of the A-phase upper bridge arm is positive is shown in Figure 4.

When the number of modulation output modules is positive, first calculate the modulation wave Uapout of the upper bridge arm of phase a according to formula (2), and then calculate the modulation wave Umap of each module as shown in formula (5). Where $\mathrm{N}$ is the number of modules in a single bridge arm, and Napb is the number of modules bypassed due to module failure.

$$
U_{\text {map }}=\frac{U_{\text {apout }}}{\left(N-N_{a p b}\right) * u_{m}}
$$

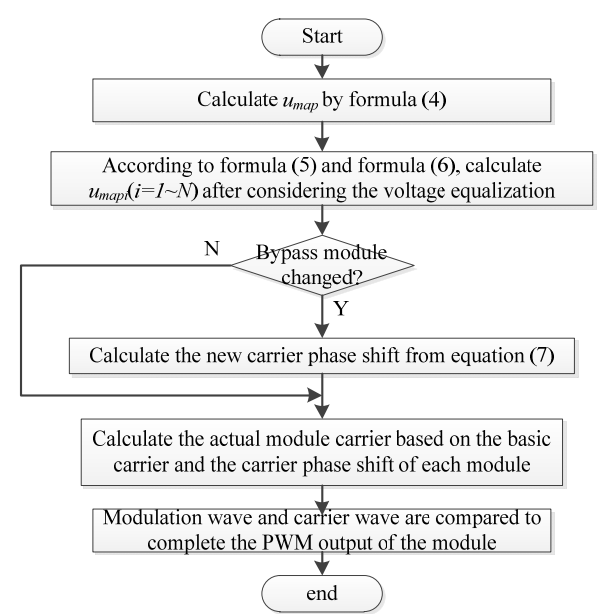

Figure4 Flow chart of CPS-PWM method

There is an independent pressure equalizing ring for each module, and the unified modulation wave superimposed on the output of the equalizing ring is used to obtain the modulation wave of each module to achieve voltage equalization. The calculation process is shown in equations (6) and (7). Among them, uapmavr is the average voltage of the non-fail-by-pass module of the upper bridge arm of phase A; napb is the serial number of the fail-by-pass module; uapmi is the module voltage of the i-th module; Umapi is the final output modulation wave of the $\mathrm{i}$-th module; $\mathrm{kp}$ is the proportional coefficient of the voltage equalizing ring. By adjusting the size of $\mathrm{kp}$, the voltage difference of the modules in a single bridge arm in operation can be adjusted.

$$
\begin{gathered}
u_{\text {apmavr }}=\frac{1}{N-N_{a p b}} \sum_{\substack{i=1 \sim N, i \neq n_{a p b}}} u_{\text {apmi }} \\
U_{\text {mapi }}=U_{\text {map }}+k_{p}\left(u_{\text {apmavr }}-u_{\text {apmi }}\right), i=1 \sim N, i \neq n_{\text {apb }}
\end{gathered}
$$

After obtaining the modulation wave of each module, the carrier of each module needs to be updated according to whether the fail-open module changes or not. The carrier uses a triangular wave, and the angle period is $2 \pi$. If the bypass module changes, the carrier phase shift of each module needs to be recalculated, as shown in the formula. $\theta \mathrm{i}$ is the carrier phase shift of the $\mathrm{i}$-th module, and the carrier phase shift of the carrier is superimposed on the basic carrier to obtain the carrier of each module.

$$
\theta_{i}=\frac{\pi}{N-N_{a p b}}(i-1), i=1 \sim N, i \neq n_{a p b}
$$

For the CPS-PWM modulation method, the carrier frequency fc is about the switching frequency of each module, and the equivalent switching frequency of the MMC AC output is about $2 \mathrm{Nfc}$; the modulation wave and carrier of each module in the bridge arm obtained by calculation are compared to obtain each PWM modulation output of the module. The above completes the modulation process of CPS-PWM.

It can be seen from the implementation of CPS-PWM modulation method. The above two calculation characteristics of CPS-PWM cause too much calculation in implementation. This has caused significant difficulties in practical applications. Therefore, it is necessary to further improve the modulation method to 
obtain a good output effect while considering the occupation of computing resources.

\section{HIGH QUALITY NLM METHOD}

Traditional NLM modulation and CPS-PWM have their own advantages and disadvantages when they are applied to low- and medium-voltage MMC with a small number of modules. By analyzing and comparing the advantages and implementation methods of the two, this paper proposes a high-quality NLM modulation method. This method has many advantages such as high equivalent switching frequency, small calculation amount, stable voltage balance, stable output highfrequency harmonic frequency, easy configuration of output filter, simple redundant configuration, and simple control of hybrid bridge MMC. Taking the A phase upper arm modulation output voltage as positive and the carrier frequency as the control frequency as an example, the modulation method flowchart is shown in Figure 5.

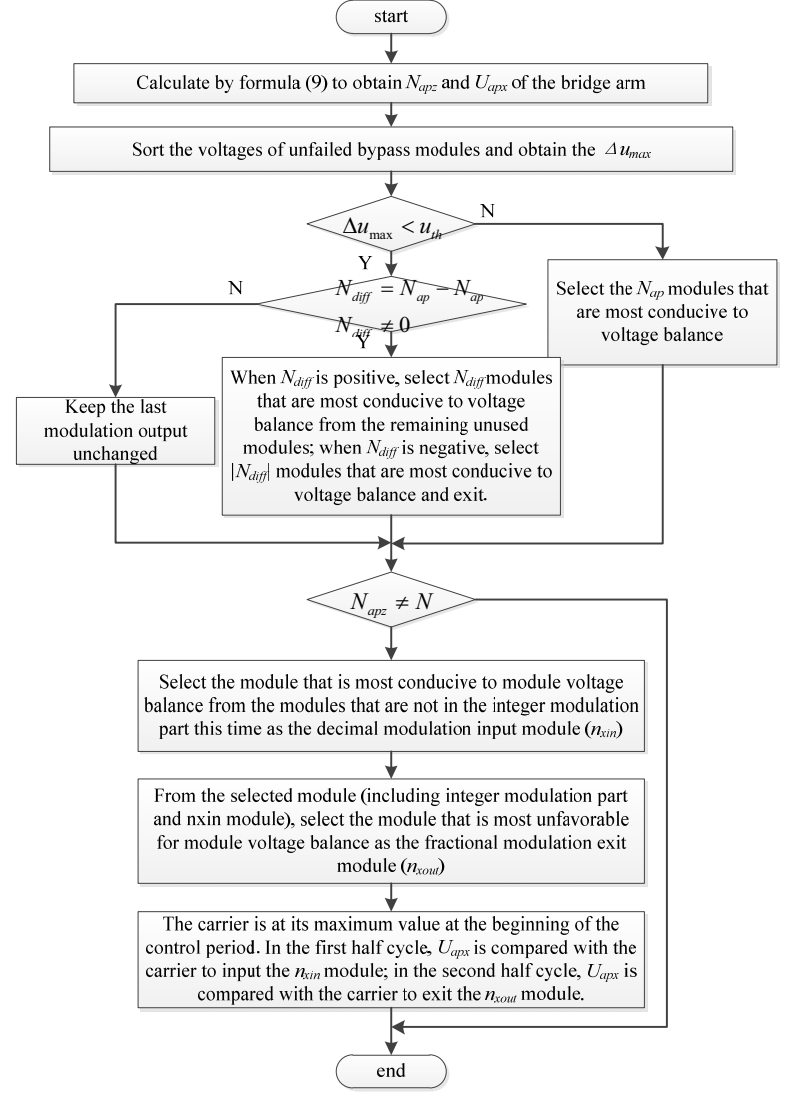

Figure 5 Flow chart of high quality NLM modulation method

The modulation process can be divided into the following steps:

1)Calculate the integer part and fractional part of the modulation output by formula (9). Among them, $N_{a p z}$ is the integer modulation part, $U_{a p x}$ is the fractional modulation part; $f i x()$ is the rounding function to zero. Through this rounding method, a module that does not have both positive and negative output levels in the bridge arm can be realized. Maximize the output effect.

$$
\left\{\begin{array}{l}
N_{a p z}=f i x\left(\frac{U_{a p o u t}}{u_{\mathrm{m}}}\right) \\
U_{a p x}=\frac{U_{a p o u t}}{u_{m}}-N_{a p z}
\end{array}\right.
$$

2)Use $N_{a p c}$ as the number of modulation output modules to execute the same sorting selection logic as NLM modulation, and complete integer modulation input module selection.

3)Judge whether the number of integer modulation modules reaches the maximum number of modules. When $N_{a p c}=N$, no decimal modulation logic will directly complete the modulation calculation output; when $N_{\text {apc }} \neq N$, the decimal modulation logic will be executed.

First, according to the sign of the bridge arm current $i_{a p}$, select the decimal input module from the module that is not in the range of the integer modulation module this time. When $i_{a p} \geq 0$, select the module with the lowest voltage; when $i_{a p}<0$, select the module with the highest voltage. $n_{x i n}$ is the serial number of the decimal input module.

Next, select the decimal exit module from the selected modules (including integer modulation part and decimal input module) according to the sign of the bridge arm current $i_{a p}$. When $i_{a p} \geq 0$, select the module with the highest voltage; when $i_{a p}<0$, Select the module with the lowest voltage. $n_{\text {xout }}$ is the serial number of the decimal exit module.

4)The last part is the output part, which is output according to the calculation result at the beginning of the next control cycle. The integer modulation part is put in at the beginning of the control period; the nxin module is put in by comparing $U_{a p x}$ with the carrier in the first half period; the $n_{\text {xout }}$ module is withdrawn by comparing $U_{a p x}$ with the carrier in the second half period.

The above is the complete process of a modulation calculation output. When the output voltage of the bridge arm is negative, all the half bridges are withdrawn, and only the full bridge is put into operation. The algorithm logic is similar to the above, so I will not repeat it.

The equivalent switching frequency of the highquality NLM modulation method is $2 f_{\text {con }}$, where $f_{\text {con }}$ is the fractional carrier frequency, and the IGBT switching frequencies of the full-bridge and half-bridge modules are shown in equation (10) respectively. The calculation does not include the additional switching frequency for voltage equalization when the module voltage difference exceeds the limit, but this part is very small.

$$
\left\{\begin{array}{l}
f_{F I G B T} \approx \frac{f_{c o n}}{2 N} \\
f_{H I G B T} \approx \frac{f_{c o n}}{N}
\end{array}\right.
$$

This modulation strategy solves the two main problems of traditional NLM modulation through the cooperative switching of two modules in each control cycle. The first is to raise the equivalent switching frequency to the level of the control period, and the switching frequency is acceptable. Secondly, the module voltage balance can be achieved through the input of modules that are beneficial to voltage equalization and 
the exit of modules that are not conducive to voltage equalization in each control cycle, which can greatly reduce the increase in switching frequency caused by module voltage equalization.

In terms of calculating pressure, this modulation method only needs to sort and select all non-bypass modules once in each control cycle, without multiplication and division calculations, and without increasing computing resources for bypass conditions. The realization of these calculation processes in FPGA is extremely convenient and the resource usage is very small. Compared with the practical application of CPSPWM control method, it has great advantages in calculating pressure.

\section{SIMULATION VERIFICATION}

Use PSCAD/EMTDC to build MMC simulation model to compare the output waveform, voltage fluctuation, harmonic frequency, switching frequency and other parameters of the above three modulation methods. The parameters of MMC are shown in Table 1. Two control methods are used to verify the modulation method, namely, the open-circuit control AC voltage mode and the closed-loop control AC current mode.

Table 1 Detailed model parameters

\begin{tabular}{|c|c|c|c|}
\hline $\begin{array}{c}\text { Rated AC } \\
\text { voltage }\end{array}$ & $5 \mathrm{kV}$ & $\begin{array}{c}\text { Bridge arm } \\
\text { inductance }\end{array}$ & $1.6 \mathrm{mH}$ \\
\hline $\begin{array}{c}\text { Rated DC } \\
\text { voltage }\end{array}$ & $\pm 5 \mathrm{kV}$ & $\begin{array}{c}\text { Module } \\
\text { capacitance }\end{array}$ & $12360 \mu \mathrm{F}$ \\
\hline Rated Capacity & $10 \mathrm{MVA}$ & $\begin{array}{c}\text { Number of full } \\
\text { bridge modules }\end{array}$ & 6 \\
\hline $\begin{array}{c}\text { Number of } \\
\text { single bridge } \\
\text { arms }\end{array}$ & 12 & $\begin{array}{c}\text { Number of half- } \\
\text { bridge modules }\end{array}$ & 6 \\
\hline $\begin{array}{c}\text { AC frequency } \\
\text { fres-PWM } \\
\text { carrier } \\
\text { frequency }\end{array}$ & $420 \mathrm{~Hz}$ \\
\hline $\begin{array}{c}\text { NLM carrier } \\
\text { frequency }\end{array}$ & $10 \mathrm{kHz}$ & & \\
\hline
\end{tabular}

\subsection{Open circuit control AC voltage}

The DC pole-to-pole voltage is $10 \mathrm{kV}$, the MMC AC side line is disconnected, the output port is open, and the outer loop output is the same, using three modulation methods to output the voltage waveform as shown in Figure 6(a). The statistics of the switching times of the full half-bridge IGBT under each modulation are shown in Figure 6(b), and the result of FFT analysis on each output waveform is shown in Figure 6(c).

It can be seen from Figure 6(b) that at this time, the CPS-PWM modulation mode and the high-quality NLM modulation mode are basically the same for the switching frequency of each IGBT of the full half bridge. The FFT analysis result in Figure 6(c) shows that the harmonic frequency of CPS-PWM is mainly $10 \mathrm{k}$, and there are a large number of harmonic components near the double frequency; the harmonic frequency of highquality NLM modulation is concentrated near $20 \mathrm{k}$. In the figure, a small amount of harmonic components around
$10 \mathrm{k}$ are caused by the control frequency. Through calculation, the THD of CPS-PWM is $8.60 \%$, and the THD of high-quality NLM is $5.08 \%$.

However, because there is no current, the NLM modulation switching frequency is very low, and the harmonic components output by the NLM are concentrated in the low frequency range, and the THD is $5.96 \%$, but it is difficult to filter due to the low frequency.

In summary, when the switching frequency of the high-quality NLM modulation method proposed in this article is consistent with CPS-PWM, the modulation output harmonic frequency is higher and the THD is lower. Compared with NLM, the THD of high-quality NLM is similar in size, but its harmonic frequency is much higher, and filtering configuration can be easier.

\subsection{Closed loop control AC current}

The voltage between the DC poles is $10 \mathrm{kV}$, the $\mathrm{AC}$ side of the MMC is connected to the grid, and the AC current is controlled. The current loop uses the deadbeat control method to control the output of the full active power of $10 \mathrm{MW}$ and suppress the circulating current to 0 .

In the case of the same outer loop output, three modulation methods are used to obtain the output current as shown in Figure 7(a). The statistics of the switching times of the full half-bridge IGBT is shown in Figure 7(b). The voltage of the upper bridge arm module of phase $a$ is shown in Figure 7(c).

Comparing Figure 6(b) and Figure 7(b), it can be seen that when the MMC emits full active power, the IGBT switching frequency of CPS-PWM and highquality NLM modulation methods does not change significantly, and the switching frequency of the two modulation methods is similar.

It can be seen from the module voltage equalization situation in Figure 7(c) that these two modulation methods have a good voltage equalization effect, and the maximum deviation of the unified bridge arm module voltage is within $20 \mathrm{~V}$. However, due to the increase of the equalizing pressure, the switching frequency of NLM modulation is significantly increased compared to the no-load VF, but it is still lower than the switching frequency of the other two modulation methods, but the corresponding voltage equalization effect is poor, and the maximum module voltage deviation is $25 \mathrm{~V}$.

From the current output in Figure 7(a), it can be seen that among the three modulation methods, the current output current quality of CPS-PWM modulation and high-quality NLM modulation is similar, the current THD under CPS-PWM modulation is $0.63 \%$, and the high-quality NLM The current THD under modulation is $0.53 \%$. However, the power quality of the NLM modulated output current is poor, and the THD is $2.01 \%$.

From the simulation comparison of the above two control situations, it can be concluded that the highquality NLM modulation proposed in this article is slightly better than CPS-PWM and far better than NLM regardless of whether it is in VF mode or controlled AC current mode. And high quality NLM modulated output 
voltage has higher harmonic frequency. High-quality NLM modulation has stable switching frequency and stable voltage equalization effect under various operating conditions. CPS-PWM modulation has the same characteristics, while traditional NLM modulation has a greater relationship between switching frequency and current. Finally, the high-quality NLM modulation proposed in this paper has slightly better effects than CPS-PWM modulation, and at the same time has smaller calculation requirements, more convenient redundancy configuration capabilities, and easier implementation of hybrid bridge modulation. This makes the modulation method can be well used in low- and medium-voltage MMC.

\section{CONCLUSION}

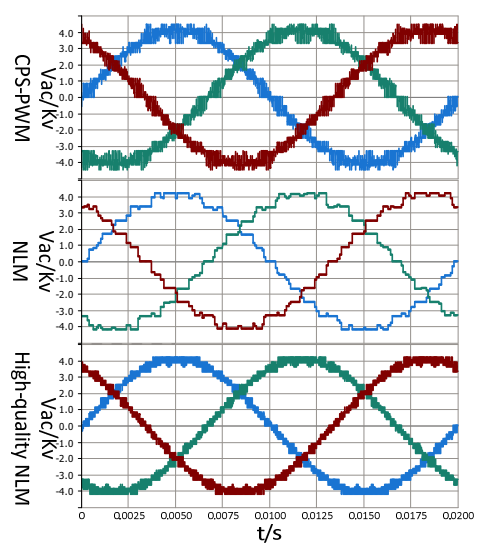

(a) AC voltage waveform

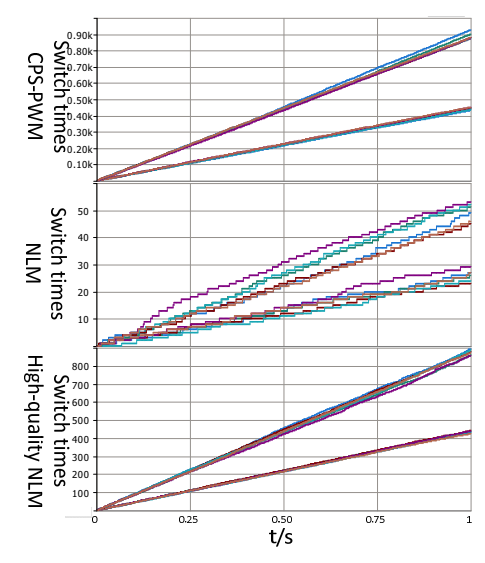

(b) IGBT switching frequency waveform
This article combines the advantages of the traditional NLM modulation method and the traditional CPS-PWM modulation method to propose a high-quality NLM modulation method. The modulation method has the advantages of two modulation methods at the same time, including simple redundant configuration, fixed switching frequency, high equivalent switching frequency, and stable voltage equalization effect. At the same time, the amount of calculation is very small, which is suitable for the practical application of medium and low voltage MMC. The PSCAD simulation software uses the same outer loop control method to compare and verify the effectiveness of the high-quality NLM modulation method proposed in this paper.
Figure 6 Comparison of three modulation outputs of open-circuit control AC voltage

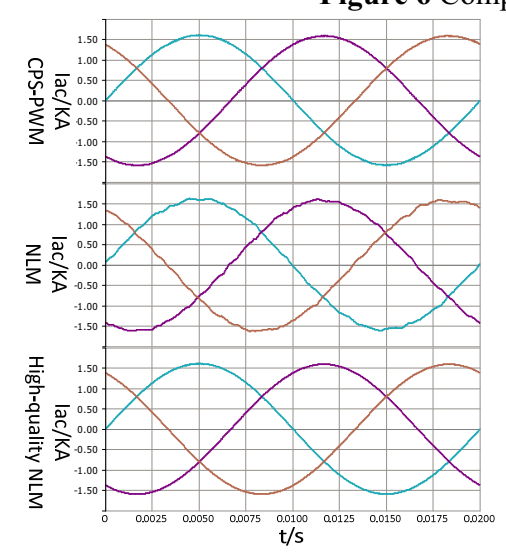

(a) AC current waveform

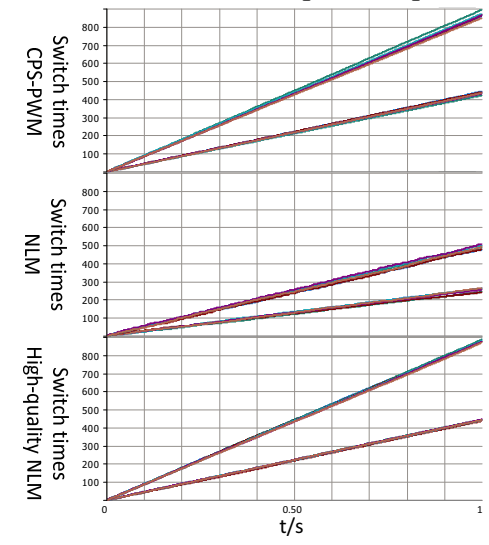

(b) IGBT switching frequency waveform

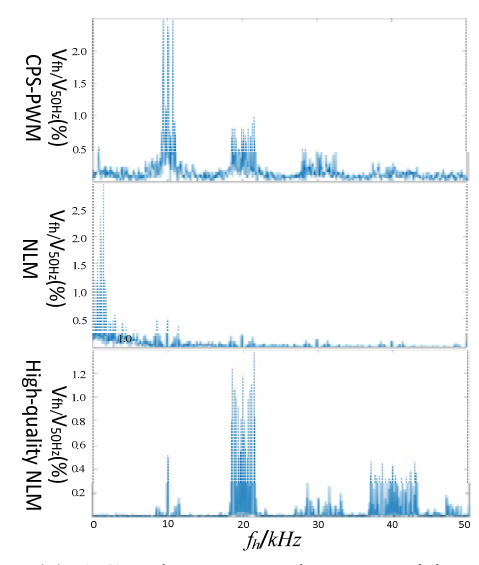

(c) AC voltage FFT decomposition spectrum
Figure 7 Comparison of three modulation outputs of closed-loop control AC current

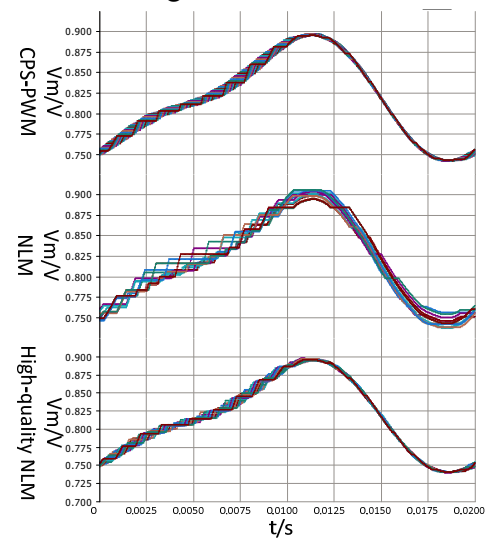

(c) A phase upper bridge arm module voltage waveform diagram
3. Huang A Q, Crow M L, Heydt G T, et al. The Future Renewable Electric Energy Delivery and Management (FREEDM) System: The Energy Internet[J]. Proceedings of the IEEE, 2011, 99(1):133-148.

4. Kakigano H, Miura Y, Ise T, et al. DC micro-grid for super high quality distribution - System configuration and control of distributed generations and energy storage devices[C]// 2006 37th IEEE Power Electronics Specialists Conference. IEEE, 2017. 
5. Kakigano H, Miura $\mathrm{Y}$, Ise $\mathrm{T}$. Low-Voltage Bipolar-Type DC Microgrid for Super High Quality Distribution[J]. IEEE Transactions on Power Electronics, 2010, 25(12):3066-3075.

6. Weiss R, Boeke U, Ott L . Energy Efficient LowVoltage DC-Grids for Commercial Buildings[C]// IEEE First International Conference on Dc Microgrids. IEEE, 2015.

7. Diaz E R, Su X, Savaghebi M, et al. Intelligent DC microgrid living laboratories - A Chinese-danish cooperation project[C]// IEEE First International Conference on Dc Microgrids. IEEE, 2015.

8. Ryu M H , Kim H S , Baek J W, et al. Effective Test Bed of 380-V DC Distribution System Using Isolated Power Converters[J]. IEEE Transactions on Industrial Electronics, 2015, 62(7):4525-4536.

9. Debnath S, Qin J , Bahrani B , et al. Operation, Control, and Applications of the Modular Multilevel Converter: A Review[J]. IEEE Transactions on Power Electronics, 2015, 30(1):37-53.

10. Perez M A, Bernet S, Rodriguez J , et al. Circuit Topologies, Modeling, Control Schemes, and Applications of Modular Multilevel Converters[J]. IEEE Transactions on Power Electronics, 2015, 30(1):4-17.

11. FranqueLo L G, Rodriguez J, Leon J I, et al. The Age of Multilevel converters arrives[J], IEEE Industrial Electronics Magazine, 2008, 2(2):28-39. 